\title{
Molecules in the disk orbiting the twin young suns of V4046 Sagittarii
}

\author{
J. H. Kastner ${ }^{1,2}$, B. Zuckerman ${ }^{3,4}$, P. Hily-Blant ${ }^{1}$, and T. Forveille ${ }^{1}$ \\ ${ }^{1}$ Laboratoire d'Astrophysique de Grenoble, Université Joseph Fourier - CNRS, BP 53, 38041 Grenoble Cedex, France \\ e-mail: joel.kastner@obs.ujf-grenoble.fr \\ 2 Chester F. Carlson Center for Imaging Science, Rochester Institute of Technology, 54 Lomb Memorial Dr., Rochester, \\ NY 14623, USA \\ e-mail: jhk@cis.rit.edu \\ 3 Dept. of Physics \& Astronomy, University of California, Los Angeles 90095, USA \\ 4 UCLA Center for Astrobiology, University of California, Los Angeles 90095, USA
}

Received 17 August 2008 / Accepted 29 September 2008

\section{ABSTRACT}

\begin{abstract}
Context. Direct information concerning the physical conditions and chemistry within the Jovian planet-building zones of circumbinary disks surrounding pre-main sequence stars is essentially nonexistent, especially for the more evolved pre-MS systems in which planets may already be forming or may have formed.

Aims. We searched for a gaseous component within the dusty circumbinary disk around the nearby ( $D \sim 70$ pc), 12 Myr-old system V4046 Sgr - a tight ( $9 R_{\odot}$ separation), short-period $\left(P \sim 2.42\right.$ day) binary with nearly equal component masses of $\sim 0.9 M_{\odot}-$ so as to assess the mass, chemistry, and kinematics of this gaseous disk.

Methods. We conducted a mm-wave molecular line survey of V4046 Sgr with the $30 \mathrm{~m}$ telescope of the Institut de Radio Astronomie Millimetrique (IRAM). We use these data to investigate the kinematics, gas mass, and chemical constituents of the V4046 Sgr disk. Results. We detected rotational transitions of ${ }^{12} \mathrm{CO}{ }^{13} \mathrm{CO}, \mathrm{HCN}, \mathrm{CN}$, and $\mathrm{HCO}^{+}$. The double-peaked CO line profiles of V4046 Sgr are well fit by a model invoking a Keplerian disk with outer radius of $\sim 250 \mathrm{AU}$ that is viewed at an inclination $i=35^{\circ}$. We infer minimum disk gas and dust masses of $\sim 13$ and $\sim 20$ Earth masses from the V4046 Sgr CO line and submm continuum fluxes, respectively. The actual disk gas mass could be much larger if the gas-phase $\mathrm{CO}$ is highly depleted and/or ${ }^{13} \mathrm{CO}$ is very optically thick.

Conclusions. The overall similarity of the circumbinary disk of V4046 Sgr to the disk orbiting the single, $~ 8$ Myr-old star TW Hya - a star/disk system often regarded as representative of the early solar nebula - indicates that gas giant planets are likely commonplace among close binary star systems. Given the relatively advanced age and proximity of V4046 Sgr, these results provide strong motivation for future high-resolution imaging designed to ascertain whether a planetary system now orbits its twin suns.
\end{abstract}

Key words. stars: circumstellar matter - stars: individual: V4046 Sgr - stars: binaries: close - stars: pre-main sequence stars: planetary systems: protoplanetary disks

\section{Introduction}

Because precision radial velocity searches for planets have thus far avoided close (separation $<1 \mathrm{AU}$ ) binary star systems, our present knowledge of the potential existence of planets orbiting such systems is limited to theoretical models of giant planet formation in circumbinary disks (Pierens \& Nelson 2008). Such models are motivated primarily by detections of disks orbiting a handful of very young (age $\lesssim 1 \mathrm{Myr}$ ) spectroscopic binaries (the best-studied case being GG Tau; Dutrey et al. 1994). However, observational constraints on the physical conditions and chemistries within the Jovian planet-building zones of circumbinary disks - particularly those disks orbiting older pre-main sequence (pre-MS) binary systems, in which planets may already be forming or may have formed - are essentially nonexistent.

V4046 Sgr is a close ( $\sim 9 R_{\odot}$ separation), 2.42 day period, pre-main sequence (pre-MS) binary with nearly equal component masses of $\sim 0.9 M_{\odot}$ that is found far from any dark cloud (Quast et al. 2000; Stempels \& Gaum 2004). Its likely membership in the $\beta$ Pic Moving Group, which has a "trace-back" age of $\sim 12$ Myr (Ortega et al. 2002; Song et al. 2003), implies a distance from Earth of $\sim 72 \mathrm{pc}$ (Torres et al. 2006, 2008). The system has substantial mid- to far-IR excess emission indicative of a "transitional" circumstellar disk, i.e., a dusty circumbinary disk with a central gap (Jenson \& Mathieu 1997). V4046 Sgr is also a luminous X-ray source and, furthermore, its X-ray spectrum reveals evidence for low-temperature, high-density plasma indicative of accretion shocks (Guenther et al. 2006).

In all of these respects - save an important one, its binarity - V4046 Sgr closely resembles the nearby, classical T Tauri star TW Hya (e.g., Kastner et al. 1997, 2002; and references therein). As a consquence of its relatively advanced age ( $\sim 8 \mathrm{Myr}$; Song et al. 2003) and proximity to Earth $(D=53.7 \mathrm{pc}$; van Leeuwen 2007), the TW Hya star-disk system has been closely scrutinized and, in particular, has served as a prototype for the study of gaseous (molecular) disks around pre-main sequence stars via mm-wave molecular spectroscopy and imaging (Kastner et al. 1997; Thi et al. 2004; Qi et al. 2004, 2006, 2008). Despite the many parallels between TW Hya and V4046 Sgr, however, no molecular line observations of the latter system have been reported. Hence, to ascertain whether the circumbinary disk orbiting V4046 Sgr possesses a detectable gaseous component, and whether the physical conditions within such a gaseous disk might be conducive to the formation of giant protoplanets, we conducted a molecular line survey of V4046 Sgr 
with the $30 \mathrm{~m}$ telescope of the Institut de Radio Astronomie Millimetrique (IRAM).

\section{Observations}

\subsection{Data acquisition and reduction}

We observed V4046 Sgr $(\mathrm{RA}=18: 14: 10.466$, $\mathrm{dec}=-32: 47: 34.50$ ) with the IRAM $30 \mathrm{~m}$ telescope (in remote mode) on 26 and 27 May 2008. The molecules and transitions observed are shown in Table 1 and Figs. 1 and 2. We observed simultaneously in either the $100 \mathrm{GHz}(3 \mathrm{~mm})$ and $230 \mathrm{GHz}(1 \mathrm{~mm})$ or $150 \mathrm{GHz}(2 \mathrm{~mm})$ and $270 \mathrm{GHz}(1 \mathrm{~mm})$ bands, and in both polarizations in each band, using receiver combinations $\mathrm{A} 100+\mathrm{B} 100$ and $\mathrm{C} 230+\mathrm{D} 230$ or $\mathrm{A} 150+\mathrm{B} 150$ and C270+D270 (all in SSB mode), respectively. For the spectral line backends, we used both the VESPA autocorrelator with $40 \mathrm{kHz}$ and $80 \mathrm{kHz} \mathrm{MHz}$ resolution and the $1 \mathrm{MHz}$ filter banks. The weather was excellent to good $\left(\tau_{225} \sim 0.05-0.3\right)$ throughout the period; time-averaged system temperatures in both the $3 \mathrm{~mm}$ and $1 \mathrm{~mm}$ bands were in the range $200-1500 \mathrm{~K}$ for this very low elevation $\left(\sim 15-20^{\circ}\right)$ source. We checked pointing and focus (using nearby quasars as references) every 1-2 h. Typical pointing errors were $\sim 3^{\prime \prime}$, i.e., $\sim 1 / 8$ beamwidth for $3 \mathrm{~mm}$ (FWHP beamwidth 21") and $\sim 1 / 4$ beamwidth for $1 \mathrm{~mm}$ (FWHP beamwidth $12^{\prime \prime}$ ). Spectra were acquired in wobbler switching mode, resulting in flat spectral baselines.

We used the $\mathrm{CLASS}^{1}$ radio spectral line data reduction package to sum all individual spectral scans obtained in both polarizations for a given transition, and then to subtract a linearfit baseline from each of these integrated spectra, calculating channel-to-channel noise levels in the process. All antenna temperature measurements reported in Table 1 (i.e., peak main-beam brightness temperature $T_{\mathrm{mb}}$ and integrated line intensity $I$; see Sect. 3.1) have been corrected assuming the beam efficiencies $\left(B_{\text {eff }}\right)$ listed in the table.

\subsection{Results}

We detected lines of ${ }^{12} \mathrm{CO}{ }^{13} \mathrm{CO}, \mathrm{HCN}, \mathrm{CN}$, and $\mathrm{HCO}^{+}$from the circumbinary disk of V4046 Sgr. Measured line parameters are reported in Table 1. For the CO lines - whose profiles are sharply double-peaked (with velocity separation $v_{\mathrm{d}}$ between the peaks) - peak and integrated line intensities ( $T_{\mathrm{mb}}$ and $I$, respectively) were determined empirically (from the peak and velocityintegrated main beam brightness temperatures, respectively), while $v_{\mathrm{d}}$ was determined from a parametric fit of a model of molecular emission from a Keplerian disk (see Sect. 3.1). It appears that the $\mathrm{HCN}$ and $\mathrm{CN}$ lines, as well as the $\mathrm{HCO}^{+}(3-2)$ line, also display double peaks at the approximate velocities of those detected in the $\mathrm{CO}$ lines. In the case of $\mathrm{CN}$ and $\mathrm{HCN}$, the line profiles include contributions from closely-space hyperfine components (e.g., Skatrud et al. 1983), which tends to "wash out" their potential double-peaked line shapes. Hence, simple singleGaussian fits served to determine these line parameters (including $\mathrm{HCO}^{+}(3-2)$, for which the signal-to-noise ratio renders a two-Gaussian fit unreliable).

Substantial asymmetries are present in the two $\mathrm{CO}(2-1)$ line profiles (and, possibly, $\mathrm{HCO}^{+}(3-2)$ ), wherein the red peak is noticeably stronger than that of the blue. This contrast, which is not apparent in the ${ }^{12} \mathrm{CO}(1-0), \mathrm{CN}$, or $\mathrm{HCN}$ lines, appears more profound than the slight difference between the red and blue

1 See http://iram.fr/IRAMFR/GILDAS/ peak intensities typical of pre-MS disk CO lines (see, e.g., Fig. 6 in Thi et al. 2004). We consider the potential origins for these $\mathrm{CO}(2-1)$ line profile asymmetries in Sect. 3.1.

\section{Analysis and discussion}

\subsection{Disk structure and kinematics}

To ascertain the kinematic characteristics of the V4046 Sgr disk, we fit the CO line profiles using the Kastner et al. (2008) parameterization of the detailed Keplerian disk model presented in Beckwith \& Sargent (1993). This model fitting exercise directly yields an estimate of the projected outer disk rotation velocity $\left(v_{\mathrm{d}}\right.$, equivalent to the half-value of the velocity separation of the red and blue peaks in the line profiles), as well as indications of the slope $(q)$ of the disk radial temperature profile $T \propto r^{-q}$ (obtained from the shape of the high- $v$ line wings) and the slope $\left(p_{\mathrm{d}}\right)$ of the inner line profile (i.e., between $v=v_{\mathrm{d}}$ and $v=0)$. In the case of an edge-on disk, $p_{\mathrm{d}}$ provides a measure of the definition of the disk outer edge (i.e., $p_{\mathrm{d}}=1$ corresponds to a sharp outer edge and values $p_{\mathrm{d}}<1$ indicate lack of a sharp edge; Kastner et al. 2008). However, in the present case of a disk that is likely viewed at intermediate inclination (see below), the central regions of the CO line profiles can be "filled in" by contributions from gas spanning a wide range of disk radii (e.g., Fig. 3 in Beckwith \& Sargent 1993), such that $p_{\mathrm{d}}$ is less straightforward to interpret.

Results obtained from these parametric fits to the CO line profiles are listed in Table 1. In Fig. 2 we display the best-fit model ${ }^{12} \mathrm{CO}(2-1)$ line profile, overlaid on the IRAM spectrum. This comparison of observed and model line profiles highlights the $\mathrm{CO}(2-1)$ line profile asymmetries mentioned in Sect. 2.2, as such asymmetries are not predicted by standard Keplerian disk models. Two potential explanations for these line profile asymmetries present themselves. First, it is possible that the disk structure is intrinsically asymmetric. Because the similarity of the ${ }^{12} \mathrm{CO}(2-1)$ and ${ }^{13} \mathrm{CO}(2-1)$ profiles appears to rule out optical depth effects as responsible for the line profile asymmetries - and it is unlikely that an azimuthal temperature gradient, if present, would be large enough to produce a measurable contrast in the red and blue peak intensities - the intrinsic asymmetry would most likely result from unequal beam filling factors for the redshifted and blueshifted emission. This in turn might suggest that, e.g., the redshifted disk has a larger opening angle than that of the blueshifted disk. Such an asymmetry could be caused by a perturbing body, such as a planet or low-mass companion star. The relative symmetry of the ${ }^{12} \mathrm{CO}(1-0)$ line profile would then also imply that such an intrinsic disk asymmetry is temperature-dependent.

A second, arguably more plausible, possibility is that the $\mathrm{CO}(2-1)$ source is marginally resolved by the $30 \mathrm{~m}$ and that the telescope was slightly mispointed. Specifically, the measured pointing errors of $\sim 3^{\prime \prime}$ (Sect. 2.1) are similar to the apparent disk radius inferred from the $\mathrm{CO}$ line profile analysis (see below). If the telescope beam were centered on the peak of redshifted disk emission - resulting in a $\sim 1 / 2$ beamwidth pointing offset for the blueshifted emission - the observed factor of $\sim 2$ contrast between red and blue peak intensities would result. Such a systematic mispointing furthermore would be consistent with the relative symmetry of the ${ }^{12} \mathrm{CO}(1-0)$ line profile since, at this frequency, the source should be unresolved and $\sim 3^{\prime \prime}$ pointing errors should be unimportant. Direct (interferometric) imaging of the molecular disk of V4046 Sgr is required to distinguish 
Table 1. Molecular emission lines measured toward V4046 Sgr.

\begin{tabular}{cccccccc}
\hline \hline Transition & $\begin{array}{c}v \\
(\mathrm{MHz})\end{array}$ & $B_{\mathrm{eff}}$ & $\begin{array}{c}T_{\mathrm{mb}} \\
(\mathrm{mK})\end{array}$ & $\begin{array}{c}I \\
\left(\mathrm{~K} \mathrm{~km} \mathrm{~s}^{-1}\right)\end{array}$ & $\begin{array}{c}v_{\mathrm{d}} \\
\left(\mathrm{km} \mathrm{s}^{-1}\right)\end{array}$ & $q$ & $p_{\mathrm{d}}$ \\
$(1)$ & $(2)$ & $(3)$ & $(4)$ & $(5)$ & $(6)$ & $(7)$ & $(8)$ \\
\hline${ }^{12} \mathrm{CO}(1-0)$ & 115270.204 & 0.74 & $398(56)$ & $1.09(0.03)$ & $1.14(0.05)$ & $0.56(0.07)$ & $0.35(0.04)$ \\
${ }^{12} \mathrm{CO}(2-1)$ & 230537.990 & 0.52 & $887(56)$ & $2.20(0.03)$ & $1.41(0.04)$ & $0.79(0.05)$ & $0.24(0.02)$ \\
${ }^{13} \mathrm{CO}(2-1)$ & 220398.686 & 0.55 & $346(30)$ & $1.15(0.02)$ & $1.6(0.1)$ & $0.80(0.08)$ & $0.26(0.04)$ \\
$\mathrm{HCO}^{+}(1-0)$ & 89188.523 & 0.77 & $91(27)$ & $0.32(0.04)$ & $6.3 *(1.3)$ & $\ldots$ & $\ldots$ \\
$\mathrm{HCO}^{+}(3-2)$ & 267557.625 & 0.45 & $251(130)$ & $1.19(0.26)$ & $4.7 *(1.0)$ & $\ldots$ & $\ldots$ \\
$\mathrm{CN}(2-1)$ & 226875.000 & 0.53 & $314(42)$ & $2.24(0.09)$ & $6.7 *(0.4)$ & $\ldots$ & $\ldots$ \\
$\mathrm{HCN}(3-2)$ & 265886.432 & 0.45 & $225(26)$ & $1.30(0.15)$ & $5.4^{*}(0.8)$ & $\ldots$ & $\ldots$ \\
$\mathrm{H}_{2} \mathrm{CO}\left(2_{21}-1_{11}\right)$ & 140839.000 & 0.70 & $<40$ & $<0.2$ & $\ldots$ & $\ldots$ & $\ldots$ \\
\hline
\end{tabular}

Notes. For CO lines, peak main-beam brightness temperature $\left(T_{\mathrm{mb}}\right)$ and integrated line intensity $(I)$ obtained empirically; one-half the difference between the red and blue peak velocities $\left(v_{\mathrm{d}}\right)$, radial temperature power law index $(q)$, and outer disk cutoff parameter $\left(p_{\mathrm{d}}\right)$ obtained from fits to disk model line profiles. For other lines, $T_{B, \max }$ and $I$ obtained from Gaussian fit to line profile. For these (Gaussian fit) lines the value of $v_{\mathrm{d}}$ (flagged by an asterisk) represents the FWHM of the best-fit Gaussian. Numbers in parentheses indicate formal $(1 \sigma)$ uncertainties in best-fit parameter values.

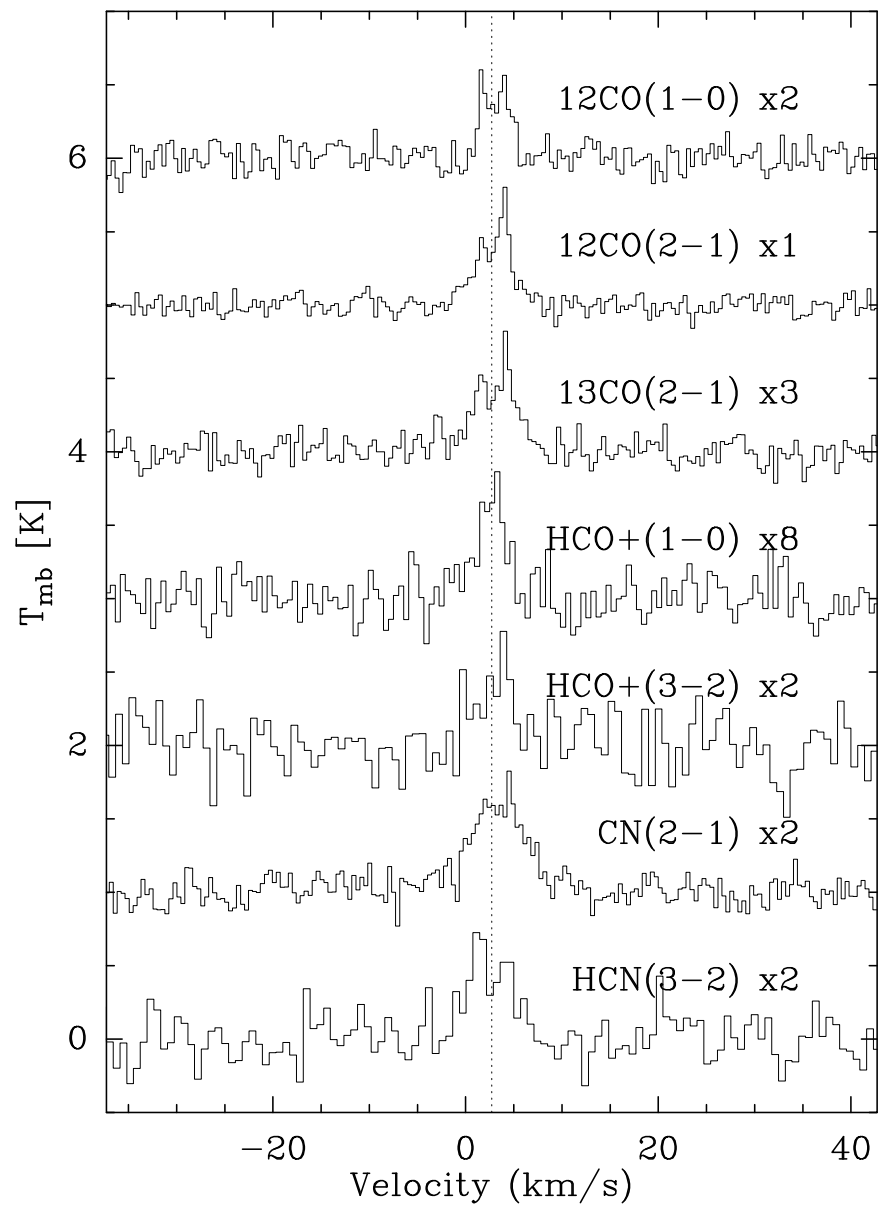

Fig. 1. Radio (mm-wave) molecular spectra of V4046 Sgr. Ordinate is velocity with respect to the Local Standard of Rest (LSR) and abscissa is main beam brightness temperature $T_{\mathrm{mb}}$. Spectral baselines are offset in $T$ for clarity. The dashed vertical line at $+2.7 \mathrm{~km} \mathrm{~s}^{-1}$ indicates the best-fit, line-center velocity of the CO lines. With this radial velocity and a distance from Earth of $72 \mathrm{pc}$, we calculate a Galactic space motion with respect to the Sun of $U=-8.0, V=-16.7, W=-7.1 \mathrm{~km} \mathrm{~s}^{-1}$.

between these alternative (intrinsic disk asymmetry vs. pointing error) explanations for the $\mathrm{CO}(2-1)$ line profile asymmetries.

Ignoring the line profile asymmetries, if one adopts the central star masses determined by Stempels \& Gahm (2004) - i.e., roughly equal components of $0.9 M_{\odot}$ - and assumes that the

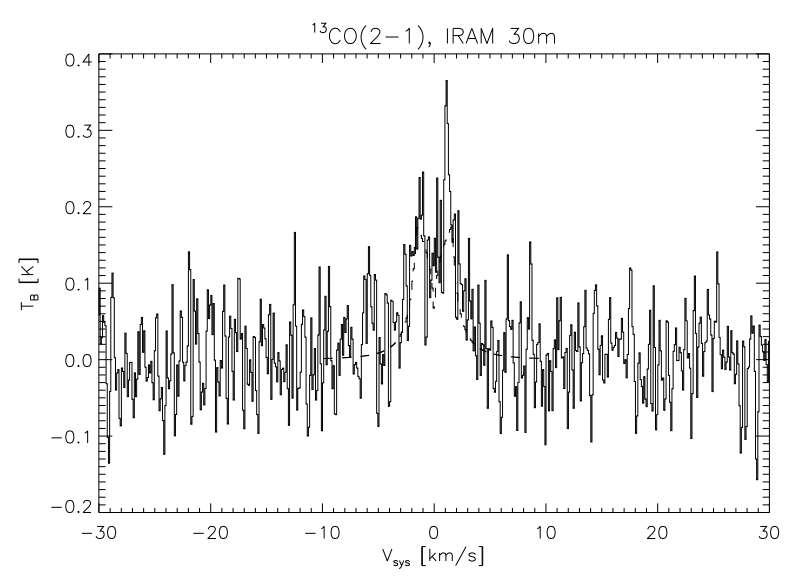

Fig. 2. ${ }^{12} \mathrm{CO}(2-1)$ line profile of V4046 Sgr (solid line) overlaid with best-fit Keplerian disk model profile (dashed line). Ordinate is velocity with respect to the systemic velocity of V4046 Sgr determined from the model fitting $\left(V_{\mathrm{LSR}}=2.7 \mathrm{~km} \mathrm{~s}^{-1}\right)$.

inclination of the disk is identical to that of the V4046 Sgr binary ( $i=35^{\circ}$, Quast et al. 2000), then the best-fit values of $v_{\mathrm{d}} \sim 1.5 \mathrm{~km} \mathrm{~s}^{-1}$ obtained from the $\mathrm{CO}(2-1)$ line profile fitting (Table 1) indicate a circumbinary disk outer radius of $\sim 250 \mathrm{AU}$. The smaller best-fit $v_{\mathrm{d}} \sim 1.1 \mathrm{~km} \mathrm{~s}^{-1}$ for the $\mathrm{CO}(1-0)$ line is indicative of a somewhat larger effective outer disk radius in this transition, consistent with its tracing colder gas at lower critical density in the outermost regions of the disk. The best-fit values of $q$ are compatible with the "canonical" range of disk temperature gradients obtained via "standard" disk models (i.e., between $q=1 / 2$ and $q=3 / 4$; e.g., Omodaka et al. 1992; Beckwith \& Sargent 1993).

An upper limit on the disk inner radius can be roughly imposed by the extent of the line wings detected in the CO line profiles. These wings are seen to extend to at least $\pm 5 \mathrm{~km} \mathrm{~s}^{-1}$, suggesting an inner radius not larger than $\sim 20 \mathrm{AU}$ for a central mass of $1.8 M_{\odot}$ and $i=35^{\circ}$. Indeed, the presence of $\sim 200 \mathrm{~K}$ dust in the disk (Sect. 3.2) indicates that the molecular disk may extend to within $\sim 3 \mathrm{AU}$ of the star.

\subsection{Disk gas and dust masses}

To estimate a lower limit on the disk gas mass from the CO line intensities, we follow the method described in Kastner et al. (2008), Zuckerman et al. (2008), and references therein. We 
adopt $D=72 \mathrm{pc}$ and a $\mathrm{CO}: \mathrm{H}_{2}$ number ratio of $10^{-4}$, and assume that the mean gas temperature in the region of the disk from which the $\mathrm{CO}$ lines originate is similar to that of the dust $(\sim 40 \mathrm{~K}$, see below). From the measured ${ }^{12} \mathrm{CO}(2-1) /{ }^{13} \mathrm{CO}(2-1)$ line ratio ( 2, Table 1), we infer that the ${ }^{12} \mathrm{CO}$ optical depth is at least $\tau_{{ }^{12} \mathrm{CO}} \sim 45$, where this minimum value is based on the assumptions that ${ }^{12} \mathrm{C} /{ }^{13} \mathrm{C}=89$ by number and that ${ }^{13} \mathrm{CO}$ is optically thin. The ${ }^{12} \mathrm{CO}$ line intensity then indicates that at least $4 \times 10^{-5} \mathrm{M}_{\odot}$ ( $\sim 13$ Earth masses) of gas resides in the circumbinary disk orbiting V4046 Sgr.

We emphasize that this CO-based estimate of the V4046 Sgr disk gas mass is likely just a stringent lower limit, because $\mathrm{CO}$ may be significantly depleted relative to $\mathrm{H}_{2}$ due to photodissociation or freezeout onto dust grains (such that $\mathrm{CO}: \mathrm{H}_{2}$ $\ll 10^{-4}$ ), and/or ${ }^{13} \mathrm{CO}$ may be optically thick. Regarding the latter possibility, the ${ }^{12} \mathrm{CO} /{ }^{13} \mathrm{CO}$ line ratio measured for V4046 Sgr is similar to that of the pre-MS molecular disk model described in Omodaka et al. (1992), who assumed a disk mass two orders of magnitude larger than the minimum mass just obtained for V4046 Sgr. Furthermore, the best-fit values of $q$ for the $\mathrm{CO}(2-1)$ lines (Table 1) are consistent with a disk temperature gradient of $T \propto r^{-3 / 4}$, characteristic of optically thick ${ }^{13} \mathrm{CO}$ emission (Beckwith \& Sargent 1993). Hence, the assumption $\tau_{{ }^{13} \mathrm{CO}}<1.0$ likely does not hold, implying the disk gas mass may be significantly larger than $\sim 13$ Earth masses. Observations of $\mathrm{C}^{18} \mathrm{O}$ would help establish $\tau_{13} \mathrm{CO}$ and thereby better constrain the mass of molecular gas orbiting V4046 Sgr.

Regardless, the V4046 Sgr disk gass mass estimated above is a factor $\sim 2$ larger than the gas mass orbiting TW Hya (after correcting for the revised distance of $45 \mathrm{pc}$ ) as estimated by Kastner et al. (1997) under the same fundamental assumptions, i.e., that $\mathrm{CO}: \mathrm{H}_{2}=10^{-4}$ and ${ }^{12} \mathrm{C} /{ }^{13} \mathrm{C}=89$ by number and $\tau^{13} \mathrm{CO}<$ 1.0. Morever, the ${ }^{12} \mathrm{CO}(2-1) /{ }^{13} \mathrm{CO}(2-1)$ line ratio measured for TW Hya $\left(\sim 7\right.$, Kastner et al. 1997) suggests that $\tau_{{ }^{13} \mathrm{CO}}<1.0$ is a better approximation in the case of TW Hya. Thus it appears that the V4046 Sgr disk likely harbors a molecular gas mass larger than that of the disk orbiting TW Hya.

Given an estimate of the dust temperature, the disk dust mass $\left(M_{\mathrm{d}}\right)$ can be estimated in the standard way (e.g., Eq. (3) in Zuckerman 2001) from the submillimeter flux of V4046 Sgr (770 mJy at $800 \mu \mathrm{m}$, Jensen et al. 1996). A spectral energy distribution (SED) comprised of IRAS and submm (Jensen et al. 1996) fluxes indicates a temperature of $\sim 40 \mathrm{~K}$ for the bulk of the dust mass, although it is clear from the SED that dust is present with a range of temperatures up to about $200 \mathrm{~K}$ (Rhee 2008 , private comm.). Adopting a dust temperature of $40 \mathrm{~K}$, then $M_{\mathrm{d}}=6 \times 10^{-5} M_{\odot}(20$ Earth masses $)$. This estimate is similar to that obtained on the basis of submm data by Webb (2000) for the TW Hya disk dust mass. If the $40 \mathrm{~K}$ grains behave like black bodies, then the orbital semi-major axis of the "cold" dust disk is $\sim 80 \mathrm{AU}$, i.e., a factor $\sim 3$ smaller than the outer disk radius obtained from the CO line profiles. This discrepancy between the inferred radii of the dust and gas disks is similar to that reported for many other pre-MS disks and is most likely an artifact of the sharp truncation of the outer disk imposed in the Keplerian disk model (Hughes et al. 2008b). It is also possible that a population of colder grains is present beyond $\sim 80 \mathrm{AU}$, however.

\section{3. $\mathrm{HCN}, \mathrm{CN}$, and $\mathrm{HCO}^{+}$: comparison with other pre-MS star/disk systems}

In Fig. 3 we plot various (sub)mm-wave molecular line ratios for V4046 Sgr, TW Hya, and five other pre-MS molecular disk systems for which emission in ${ }^{13} \mathrm{CO}, \mathrm{CN}, \mathrm{HCN}$, and $\mathrm{HCO}^{+}$have
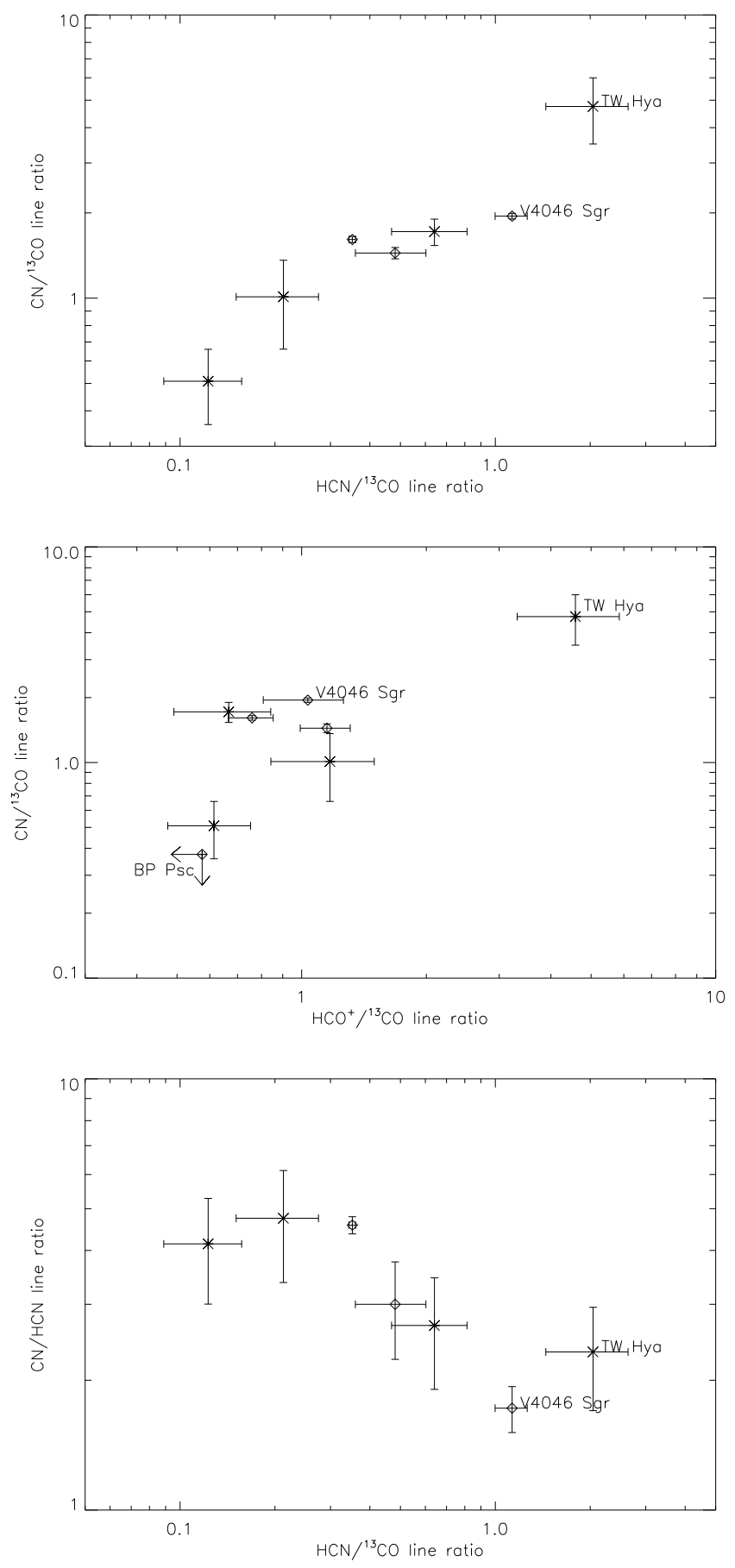

Fig. 3. $\mathrm{CN} /{ }^{13} \mathrm{CO}$ vs. $\mathrm{HCN} /{ }^{13} \mathrm{CO}$ line ratios (top), $\mathrm{CN} /{ }^{13} \mathrm{CO}$ vs. $\mathrm{HCO}^{+} /{ }^{13} \mathrm{CO}$ line ratios (middle), and $\mathrm{CN} / \mathrm{HCN}$ vs. $\mathrm{HCN} /{ }^{13} \mathrm{CO}$ line ratios (bottom) for V4046 Sgr and for the (6) other pre-main sequence stars measured to date in these lines, including the single star TW Hya (in the middle panel, we also include the unusual dusty field star BP Psc, which may be a post-MS object; see Kastner et al. 2008, and references therein). Data points corresponding to JCMT $15 \mathrm{~m}$ telescope measurements of the integrated line intensities in the $\mathrm{HCO}^{+}(4-3), \mathrm{HCN}(4-$ $3), \mathrm{CN}(3-2)$, and ${ }^{13} \mathrm{CO}(3-2)$ transitions are indicated with " $\mathrm{X}$ "; points corresponding to IRAM $30 \mathrm{~m}$ telescope measurements of the integrated line intensities in the $\mathrm{HCO}^{+}(3-2), \mathrm{HCN}(3-2), \mathrm{CN}(2-1)$, and ${ }^{13} \mathrm{CO}(2-1)$ transitions are indicated with diamonds.

all been measured (Kastner et al. 2008, and references therein). Note that the line ratio diagrams in Fig. 3 compare results from multiple transitions of these species. Hence - while systematic 
errors due to instrumental effects may be negligible (Kastner et al. 2008) - optical depth effects and departures from LTE (especially in the case of the CN lines; van Zadelhoff et al. 2003) represent potential complications when attempting to compare the line ratios measured for the various disks. That caveat aside Fig. 3 indicates that V4046 Sgr lies at the upper range of preMS star/disk systems in terms of its $\mathrm{CN}$ and $\mathrm{HCN}$ emission line strengths relative to ${ }^{13} \mathrm{CO}$, with only TW Hya displaying larger $\mathrm{HCN} /{ }^{13} \mathrm{CO}$ and $\mathrm{CN} /{ }^{13} \mathrm{CO}$ ratios. However, TW Hya still stands alone as a much stronger $\mathrm{HCO}^{+}$source (relative to ${ }^{13} \mathrm{CO}$ ) in comparison to V4046 Sgr and all other pre-MS star/disk systems.

The large relative intensities of emission lines of $\mathrm{CN}$ and $\mathrm{HCO}^{+}$, accompanying strong $\mathrm{HCN}$ emission, indicate that highenergy (UV and X-ray) radiation originating with the central stars and/or star-disk interactions has significantly and similarly impacted the chemistries of the V4046 Sgr and TW Hya disk systems (Lepp \& Dalgarno 1996; Thi et al. 2004; Kastner et al. 2008, and references therein). Models of X-ray ionization of molecular gas predict monotonically increasing $\mathrm{CN}, \mathrm{HCN}$, and $\mathrm{HCO}^{+}$abundances at constant $\mathrm{CO}$ abundance as X-ray ionization rate increases (see Figs. 2, 3 in Lepp \& Dalgarno), whereas increasing UV photodissociation rate tends to drive up the CN/HCN ratio (e.g., van Zadelhoff et al. 2003; Boger \& Sternberg 2005). It is therefore intriguing that the $\mathrm{CN} / \mathrm{HCN}$ ratios measured for the two $\sim 10$ Myr-old classical T Tauri stars are similar to those of much younger pre-MS stars even though their $\mathrm{HCN} /{ }^{13} \mathrm{CO}$ and $\mathrm{CN} /{ }^{13} \mathrm{CO}$ line ratios are significantly larger than those of the other pre-MS stars (by factors of up to $\sim 10$ for V4046 Sgr and 20 for TW Hya, Fig. 3). Given these results, and the fact that the X-ray luminosities of TW Hya and V4046 Sgr are at least a factor $\sim 3-10$ larger than those of the other pre-MS stars plotted in Fig. 3 (see Kastner et al. 2002, 2008; Guenther et al. 2006), one might speculate that X-ray ionization - as opposed to UV photodissociation - is driving the circumstellar disk chemistry in these two older TTS star-disk systems. However, it is clearly premature to conclude as much, in advance of detailed radiative transfer calculations (along the lines of those described in, e.g., van Zadelhoff et al. 2003) for all of the disks included in Fig. 3. Indeed, given a model in which X-ray ionization dominates over UV photodissociation in both disks, one must explain why the $\mathrm{HCO}^{+} /{ }^{13} \mathrm{CO}$ ratio of V4046 Sgr is much smaller than that of TW Hya, and is instead similar to those measured for younger pre-MS systems.

\section{Conclusions}

The detections of circumstellar $\mathrm{CO}, \mathrm{HCN}, \mathrm{CN}$ and $\mathrm{HCO}^{+}$presented here demonstrate that the close binary star V4046 Sgr is orbited by a rich molecular disk. At an age of $12 \mathrm{Myr}$, V4046 Sgr may be the oldest known classical $\mathrm{T}$ Tauri star and, at a distance from Earth of only $\sim 72 \mathrm{pc}$, it is also the second closest (after TW Hya). The only other star comparably close to Earth as TW Hya and V4046 Sgr and known to possess a substantial orbiting molecular disk is the A-type star 49 Cet (Zuckerman et al. 1995; Hughes et al. 2008a). Given the many young stars known to reside within 100 pc of Earth (Torres et al. 2008), it remains a mystery why these 3 stars, which span a spectral type range from $\mathrm{K} 7$ to $\mathrm{A} 1$, should have been able to retain so much orbiting molecular gas for so long (49 Cet is of uncertain age, but it may be older than V4046 Sgr).
The similarity of the composition of the molecular circumbinary disk orbiting the $\sim 12$ Myr-old, close binary V4046 Sgr to that of the molecular disk orbiting the single, $\sim 8$ Myr-old star TW Hya (Sect. 3.3) - and the likelihood that the disk gas mass of V4046 Sgr is, if anything, even larger than that of TW Hya (Sect. 3.2) - indicates that the early evolution of circumbinary disks can proceed in a manner closely analogous to that of single-star disks. Furthermore, if the TW Hya disk is indeed an appropriate system to serve as analog to the solar nebula just at or after the epoch of Jovian planet formation, then the many parallels between V4046 Sgr and TW Hya would indicate that gas giant planets are commonplace among close binary star systems. Given the age and proximity of V4046 Sgr, the results presented here therefore provide strong motivation for future high-resolution imaging designed to ascertain whether a planetary system now orbits its twin suns.

Acknowledgements. We thank Joseph Rhee for his analysis of the SED of V4046 Sgr and the anonymous referee for useful comments. J.H.K. thanks the staff of the Laboratoire d'Astrophysique de Grenoble for their support and hospitality during his yearlong sabbatical visit to that institution. This research was partially supported by a NASA grant to UCLA.

\section{References}

Beckwith, S., \& Sargent, A. 1993, ApJ, 402, 280

Boger, G. I., \& Sternberg, A. 2005, ApJ, 632, 302

Dutrey, A., Guilloteau, S., \& Simon, M. 1994, A\&A, 286, 149

Dutrey, A., Guilloteau, S., \& Guelin, M. 1997, A\&A, 317, L55

Guenther, H. M., Liefke, C., Schmitt, J. H. M. M., Robrade, J., \& Ness, J.-U. 2006, A\&A, 459, L29

Hughes, A. M., Wilner, D. J., Kamp, I., \& Hogerheijde, M. R. 2008a, ApJ, 681, 626

Hughes, A. M., Wilner, D. J., Qi, C., \& Hogerheijde, M. R. 2008b, ApJ, 678, 1119

Jenson, E., \& Mathieu, R. D. 1997, AJ, 114, 301

Jenson, E., Mathieu, R. D., \& Fuller, G. 1996, ApJ, 458, 312

Kastner, J. H., Zuckerman, B., Weintraub, D. A., \& Forveille, T. D. A. 1997, Science, 277, 67

Kastner, J. H., Huenemoerder, D. P., Schulz, N., Canizares, C. R., \& Weintraub, D. A. 2002, ApJ, 567, 434

Kastner, J. H., Zuckerman, B., \& Forveille, T. 2008, A\&A, 486, 239

Omodaka, T., Kitamura, Y., \& Kawazoe, E. 1992, ApJ, 396, L87

Ortega, V. G., de la Reza, R., Jilinski, E., \& Bazzanella, B. 2002, ApJ, 575, L75

Pierens, A., \& Nelson, R. P. 2008, A\&A, 483, 633

Qi, C., Ho, P. T. P., Wilner, D. J., et al. 2004, ApJ, 616, L11

Qi, C., Wilner, D. J., Calvet, N., et al. 2006, ApJ, 636, L157

Qi, C., Wilner, D. J., Aikawa, Y., et al. 2008, ApJ, 681, 1396

Quast, G. R., Torres, C. A. O., de La Reza, R., da Silva, L., \& Mayor, M. 2000, in Proc. IAU Symp., 200, 28

Skatrud, D. D., De Lucia, F. C., Blake, G. A., \& Sastry, K. V. L. N. 1983, J. Mol. Spec., 99, 35

Song, I., Zuckerman, B., \& Bessell, M. S. 2003, ApJ, 599, 342

Stempels, H. C., \& Gahm, G. F. 2004, A\&A, 421, 1159

Thi, W.-F., van Zadelhoff, G.-J., \& van Dishoeck, E. F. 2004, A\&A, 425, 955

Torres, C. A. O., Quast, G. R., da Silva, L., et al. 2006, A\&A, 460, 695

Torres, C. A. O., Quast, G. R., Melo, C. H. F., \& Sterzik, M. F. 2008, in

Handbook of Star Forming Regions, ed. B. Reipurth (San Francisco: Astron.

Soc. Pacific), in prep.

van Leeuwen, F. 2007, A\&A, 474, 653

van Zadelhoff, G.-J., Aikawa, Y., Hogerheijde, M. R., \& van Dishoeck, E. F. 2003, A\&A, 397, 789

Webb, R. 2000, Ph.D. Thesis, UCLA

Zuckerman, B. 2001, ARA\&A, 39, 549

Zuckerman, B., Forveille, T., \& Kastner, J. H. 1995, Nature, 373, 494

Zuckerman, B., et al. 2008, ApJ, in press [arXiv:0802 .0226] 